\title{
A Model for Accelerating Identification and Regulatory Approval of Effective Investigational Agents
}

Laura J.. Esserman, Anna D.. Barker, Janet Woodcock, Meredith A. Buxton, Donald A. Berry, Robert Patterson, Michael King. Jolly, Angela DeMichele, Nola Hylton, Eric H.. Rubin, David Parkinson, David Wholley, Laura van't Veer, Douglas Yee, John Park, Debu Tripathy, Jane Perlmutter, Kenneth Buetow, Mike Hogarth, Joe Gray, David M.. Dilts

\begin{abstract}
A path for accelerated regulatory review of new drug and biomarker combinations is badly needed to transform the current clinical drug development process into an efficient, effective system that meets current and future healthcare needs. However, this type of radical transformation will not occur by layering regulatory change on existing clinical practice patterns. Here, we summarize a May 2011 I-SPY 2 TRIAL (Investigating Serial Studies to Predict Your Therapeutic Response with Imaging and Molecular AnaLysis) workshop that included key leaders from the Food and Drug Administration (FDA), academia, industry, advocacy, and clinical trial-focused foundations that addressed this critical need. The workshop identified key aspects of the organization, trial design, and regulatory and industry alignment required to rapidly move successful agents from trial to clinical care. The workshop specifically focused on providing input for the development of a new evidence-based regulatory path for review of drugs and biomarkers that derive from neoadjuvant, modular, adaptive phase 2 screening trials achieved through precompetitive collaborations. The workshop participants agreed that new models, exemplified by the I-SPY 2 TRIAL for breast cancer, could address the need to more efficiently review and advance new drug and biomarker combinations. A three-tier model for trial development best describes this process. As practiced in I SPY 2, integrating imaging and biomarker information obtained through adaptive trials in the neoadjuvant breast cancer care setting is the first step. The second tier is replicable structural processes, including real-time data collection. The third is partner alignment achieved through pre-competitive collaboration and the potential to position successful drugs for accelerated regulatory approval in the neoadjuvant setting, where patients are likely to have the greatest benefit. Many elements of this model were incorporated into a new FDA draft guidance document which was released for public comment on May 31, 2012.
\end{abstract}

Review began 11/17/2012 Published 12/08/2012

() Copyright 2012 Esserman et al. This is an open access article distributed under the terms of the Creative Commons Attribution License CC-BY 3.0., which permits unrestricted use, distribution, and reproduction in any medium, provided the original author and source are credited.
Categories: Miscellaneous, Oncology

Keywords: drug development, accelerated regulatory review, breast cancer, nih, molecular analysis, i-spy 2, fda

\section{Introduction And Background}

In 2010, U.S. healthcare costs were $\$ 2.6$ trillion. The Center for Medicare and Medicaid Services projects that by 2020 , this economic burden will rise to $\$ 4.6$ trillion (19.8\% of the U.S. GDP [1]). An increasing share of this economic burden reflects the rising cost of drugs, with cancer drugs being a major contributor [2]. There is justifiable concern that, in the near future, new drugs may not be broadly affordable [3].

Concomitant with the rising cost of existing drugs, the development of new cancer drugs is becoming even more expensive, partly because among all drug classes, oncology drugs have the highest clinical failure rate [4]. The current return on capital investment in drug development was recently reported to be less than $0.3 \%$ - with oncology drugs providing the lowest rate of return [1]. A low probability of success and rapidly rising development costs means that drug development is increasingly the purview of only a few large companies and small, venture-backed start- up enterprises. Unfortunately, because the cost of developing a successful new drug is $\$ 1.0-1.8$ billion $[5,6]$, the investment community increasingly views financing oncology-drug development as too risky for the capital required.

Unquestionably, a significant factor that contributes to this uncertainty is the current lack of an efficient and effective system to evaluate and move new agents (or stop ineffective agents) from early clinical testing into routine clinical use. Currently, clinical drug development is fragmented, fraught with redundancies, and generally driven by traditional practices [7]. Contributing to this problem is a culture that perpetuates and rewards nonsystematic and "siloed" research approaches in both the academic and private sectors. Investigational new drug (IND) trials are evaluated in a traditional, sequential, trial-and-error progression that moves INDs, one at a time, through a series of trials, each of which is typically designed to solely evaluate a specific candidate drug (Figure 1A) [8]. The current 10-20 year development cycle for oncology drugs includes testing for safety and efficacy in the metastatic setting, followed by evaluation in the secondary prevention or adjuvant setting, and it can require several years to open, accrue and determine 
outcomes for these trials [7]. Further complicating the drug development crisis is the current tsunami of molecular data that is accumulating at a rate (i.e., doubling every six months) that exceeds Moore's Law (i.e., doubling every 18-24 months).

A. Current Development Pathway

Metastatic Setting

AdJuVANT SETting

\begin{tabular}{|c|c|c|c|c|c|c|}
\hline \multirow[b]{2}{*}{$\begin{array}{l}\text { PHASE } \\
1 \& \text { 1B }\end{array}$} & \multirow[b]{2}{*}{ PHASE 2} & \multirow[b]{2}{*}{ Phase 3} & \multirow{2}{*}{ 궁 } & \multirow{2}{*}{$\begin{array}{l}\text { 읗 } \\
\frac{5}{0}\end{array}$} & \multicolumn{2}{|c|}{ PHASE 3} \\
\hline & & & & & ACCRUAL & FOLLOW-UP \\
\hline \multicolumn{7}{|l|}{ Years } \\
\hline 0 & 2 & 6 & & & 10 & 12 \\
\hline
\end{tabular}

B. Development Pathway

Metastatic Setting

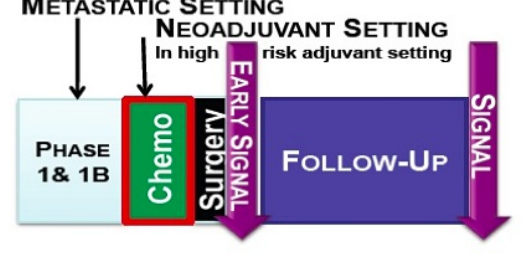

\section{FIGURE 1: Pathway diagrams}

A. Drug development traditionally begins with phase 1-3 trials in the metastatic setting; after approval in the metastatic setting, drugs move to the adjuvant setting, which requires several years for trial development and accrual. Since chemotherapy and new agents are given after surgical excision, there is a current minimum required of five years for follow-up - effectively a 10-20 year drug development cycle. B. In contrast, moving to a phase 2 screening process in the neoadjuvant setting (once drug safety is established), and selecting patients at risk for progression in the first five years after diagnosis, allows investigators to use pathologic complete response (pCR) to identify promising agents. Confirmatory testing can then be performed in the subsets of patients where the treatment benefit was greatest. This approach, used in the I-SPY 2 Trial process, is a new and promising path for shortening the clinical trials portion of the drug development cycle.

It is common practice to transition investigational cancer drugs from a small phase 2 trial to large phase 3 trials, without determining how the drugs perform against subtypes of a targeted tumor [9]. This practice is partially responsible for the failure of $66 \%$ of phase 3 cancer trials [10]. The cost of such failures is staggering, both in terms of exposing trial participants to ineffective, toxic therapy and lost investments [4]. The economic burden is further amplified by the small number of drugs that can be tested using a linear approach, which is further compounded by the opportunity costs associated with delaying or abandoning other therapeutic candidates.

Although the complex drug development crisis is multifactorial, approaches to address the problem are often too narrow. It is common to attribute the problem to the requirements of regulatory agencies, but USA regulatory standards for cancer drug safety and effectiveness are accepted worldwide and are generally faster than the regulatory approval process in Europe [11]. In the US, the median regulatory review time for cancer drugs is six months, which accounts for a small percentage of total development time [11].

\section{Knowledge turns}

One way to approach the problem is to increase "knowledge turns". Comparing development cycles in health care and the semiconductor industry, Andy Grove, $\mathrm{PhD}$, former $\mathrm{CEO}$ and co-founder of Intel, defined a knowledge turn as the time required to move from hypothesis to result, to a new hypothesis and a new result [12]. Development in the semiconductor industry relies on rapid knowledge turns and early indicators of success or failure in every experiment designed to produce a new or improved product, which is why Moore's law has been so successful in predicting the advances in semiconductors.

In oncology, a knowledge turn is essentially the time required to transit from a proposed treatment, to the evaluation of the trial result, to the development of a new treatment and new result. While knowledge turns in the semiconductor industry are measured in months, the knowledge turns in oncology drug development require years - sometimes decades. The prolonged development times in oncology drug development are caused by several factors, ranging from trial initiation, to the time needed to create and run a trial, to the outcome measurements of the trial. For example, the use of disease-free survival as an endpoint in adjuvant therapy trials necessarily translates into ten years or more for a knowledge turn. Also, cancer (unlike silicon chips), is heterogeneous, dynamic, adaptive, and subject to evolutionary pressures. However, as heterogeneity is measured more precisely, and organ-specific subtypes and pathways are better defined, knowledge turns may accelerate - and could be even further enabled through early indicators of success (or 


\section{Cureus}

failure) through the use of biomarkers to measure clinical impact. Moreover, uncovering this heterogeneity can make previously planned trials of little clinical relevance even before their results are fully reported.

\section{Solutions}

Are there solutions to this seemingly intractable problem that would ensure patient safety, guarantee more constancy in the process, and reduce knowledge-turn times? One approach, described here, is based on a workshop held in May, 2011, in which leaders from the Food and Drug Administration (FDA), academia, industry, advocacy, and clinical trial-focused foundations identified key aspects of the I-SPY 2 TRIAL (Investigating Serial Studies to Predict Your Therapeutic Response with Imaging and molecular AnaLysis) for breast cancer that could accelerate knowledge generation and rapidly move successful agents from trial to clinical care. The three-tier model proposed here is also the basis for a recently released, derivative regulatory review process, formalized as draft guidance by the FDA [13].

\section{Review}

\section{The I-Spy 2 TRIAL: a model to accelerate identification and approval of effective oncology drugs}

The I-SPY 2 TRIAL is an adaptive phase 2 trial designed to improve treatments for breast cancer patients and to accomplish the following: (1) create a new pathway to rapidly identify, confirm and ultimately gain regulatory approval for effective drugs and companion diagnostics (predictive biomarkers) for the adjuvant/neoadjuvant cancer setting; and (2) design an efficient system that explicitly addresses disease heterogeneity and can be replicated in other diseases. This novel approach could significantly reduce development time and costs for new drugs by helping to identify those patients who will benefit most from new treatments. (See Appendix for additiona details regarding the I-SPY 2 TRIAL)

\section{Organizational framework}

Rather than focusing on how each stakeholder in the drug development process acts individually, the I-SPY 2 TRIAL leverages collaboration to engender data sharing and knowledge generation that significantly accelerates learning. I-SPY 2 was designed to incorporate all of the relevant parties involved in the trial into a single group. I-SPY 2 rapidly integrates and adapts to new knowledge from patients who are on specific test drugs in order to better chose and evaluate subsequent patients who receive the same or similar drugs. The three-tier model (Figure 2, described below), is representative of a clinical research learning system that positions successful drugs for accelerated regulatory approval in the adjuvant setting. The model has direct relevance for other cancers and disease types.

\section{The adaptive trial approach used in the I-SPY 2 TRIAL consists of three tiers (Figure 2):}

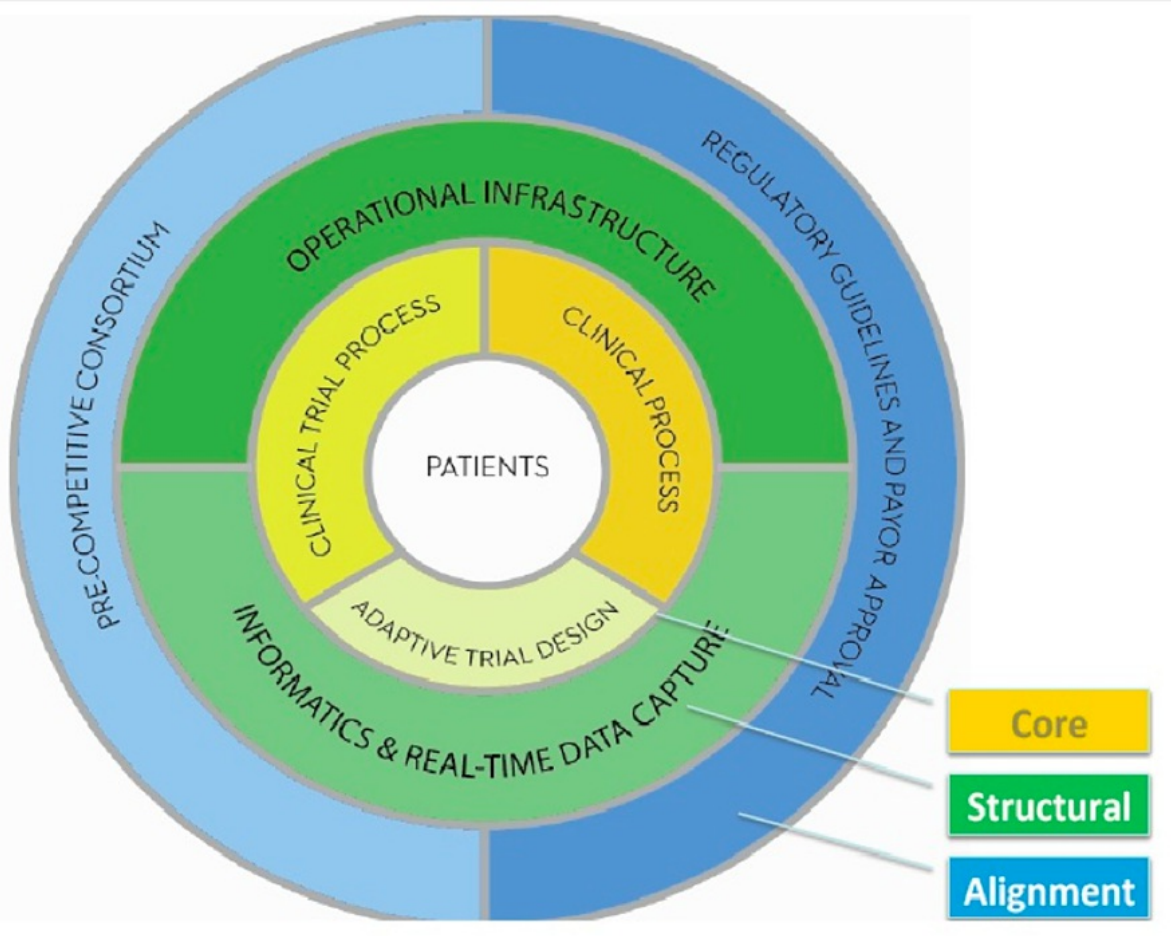




\section{FIGURE 2: Three tier model}

The three-tier model is composed of a core tier of processes (yellow) to engineer clinical care processes so that they are integrated with a modular clinical trial process that is driven by adaptive design. These concepts will need to be specific to the disease under study. The second tier of the model (green) is the set of new structural processes required to support the core tier. The third tier, the alignment process (blue), enables more efficient learning and lowers cost of development through precompetitive collaboration and pre-established rules for more accelerated approval of successful agent/biomarker pairs. This outer tier also anticipates the organizational efficiencies of linked trial phases that can accelerate the process of moving agents from safety, to screening, to confirmatory trials.

1. Core Tier, includes processes based on general principles with the goal to maximize learning and have the trial designed to adaptively optimize care. The key processes are:

a) Organization of the clinical care process to ensure that biomarkers are acquired and early treatment endpoints of efficacy are measured as a routine of care, early in the disease process. For example, giving chemotherapy before surgical excision allows tumor samples to be acquired as a routine of care before and after systemic treatment, integration of biomarkers to classify tumor, and methods for evaluating tumor response early in the course of care.

b) Use of a modularly designed trial incorporated into the backbone of standard therapy.

c) Use of an adaptive trial design constructed with the intent to accrue across biologic types, using, if possible, the majority of patients. The trial design sets rules for treatment assignment based on early endpoints to ensure real-time learning about response in the context of biomarkers that stratify the disease by subtype.

2. Structural Tier, consists of processes that are disease independent and incorporates critical organizational and structural components that support the core processes:

a) An integrated informatics platform and electronic data-capture system that enables real-time data submission and verification, and provides a randomization engine to support adaptive trial design. The emergent integrated database supports data sharing related to outcomes and biomarker relationships.

b) Organizational processes redesigned to allow for a faster and greater degree of multi-institutional cooperation. Success depends on standards for contracting, data sharing, and IP issues, and the ability to rapidly expand to other areas of critical collaboration.

3. Alignment Tier, consists of processes that are replicable across disease types (although stakeholders may differ) and involve aligning the interests of all parties to accelerate drug testing, approval, and successful use. This tier includes:

a) A new generation of collaborative consortia that promote knowledge sharing and learning across industry, academia, and regulatory agencies in a pre-competitive environment.

b) Linking of phases of agent development and testing to minimize delays. This process is analogous to a "relay race" where success is not based solely on "the speed of one leg," but also on an efficient hand-off.

c) Regulatory guidance and payer approval processes that facilitate rapid review of new treatments by these two primary gate-keepers.

Together, these tiers provide a new foundation for a model that enables highly effective agents to be identified, approved, and delivered sooner to the specific patients who are most likely to benefit.

\section{Evidence of the model's success}

The I-SPY 2 TRIAL approach has made it possible to test the elements of the proposed three-tier model. Specifically, I-SPY 2 applies the elements of the core tier, by using the neoadjuvant setting, integrating the collection and analysis of imaging and biomarkers in the course of care, and generating expression arrays as a condition of adaptive randomization to new agents. Only the biologically high-risk patients go on to be randomized. In terms of the structural tier, I-SPY 2 tested the integration of clinical and molecular data capture tools, using an interface engine (MIRTH, Mirthcorp.com), and tools developed by the National Cancer Institute's Cancer Bioinformatics Grid (caBIG). In addition, a modular adaptive randomization engine and a system to capture clinical data at the point of care were developed, which together form the 
backbone of an adaptive trials system (TRANslational informatics System to Coordinate Emerging biomarkers, Novel agents, and clinical Data, or TRANSCEND). New processes for training sites to collect and verify real-time data were instituted and plans for electronic data capture considered as primary source data by the FDA are underway. Finally, additional processes, such as a master IND, allow multiple drugs from multiple companies to be in the trial simultaneously. The trial protocol is set, and new drugs are introduced modularly through amendments, avoiding disruption to the trial as drugs flow in and out of the study. I-SPY 2 investigators have also been involved in phase 1 testing and in linking phases of drug testing. If a company goal is to get an agent into I-SPY 2, the phase lb trial can be designed using the same chemotherapy backbone, and the contract and planning process for drug entry into an I-SPY 2-like trial will be completed by the time the phase 1 trial closes. Eight agents or agent combinations have seamlessly been added into the trial and 305 patients have been successfully randomized. The ability to work on the trial development process collaboratively has led to a better understanding of the needs of all stakeholders, and provided input for the development of FDA guidance for accelerated approval of new agents in the neoadjuvant setting for early breast cancer [9].

\section{Pre-competitive consortium linking multiple stakeholders}

One major cause of the current drug development problem is the use of multiple processes among many stakeholders with minimal overlap of their respective priorities. The I-SPY 2 TRIAL addressed this problem by acknowledging up front that all primary stakeholders must be aligned before the trial began.

The rules for engagement among all stakeholders, including patient advocates, were developed collaboratively at a series of retreats. A contract "term sheet" was developed to summarize the "rules" so that companies that wished to join and add molecules to test in the I SPY 2 TRIAL process could determine quickly if they could abide by these rules before investing time in developing detailed plans for putting a drug in the trial.

In terms of the alignment tier of I SPY 2 model then, the consortium can efficiently align early in the process to provide critical information about the potential success or failure of targeted agents - and no single organization gains a competitive advantage. For example, early data availability on resistance mechanisms and diagnostic markers for targeted therapies can substantially benefit multiple drug developers and the entire scientific community. In addition, the I-SPY 2 model can eliminate the requirement for every company and academic partner to "re-invent the wheel" with respect to contracts, acceptable markers, metrics, data, and other information acceptable to regulatory agencies, effectively reducing the time and associated costs required to develop a new drug. For example, nearly identical contracts were negotiated with all participating sites and pharmaceutical and diagnostic companies, which has eliminated the need for any further negotiation as new drugs or biomarkers enter the trial. Moreover, sharing failures allows for the rapid screening out of non-beneficial agents, thereby allowing greater emphasis on more promising paths. This concept is based on what in business would be considered fast, frugal failures that quickly stops the use of limited resources in unproductive areas.

The precompetitive model also prohibits any single organization from derailing the process. For example, most clinical trials have a single primary funder, who can immediately impact the trial if resources are withdrawn. The I-SPY 2 precompetitive collaboration currently involves eight pharmaceutical companies whose drugs are tested by class, with all information shared within six months of the drug dropping or graduating from the trial. The collaboration is hosted by the Foundation for the National Institutes of Health, which serves as the honest broker for new knowledge generated in the course of the trial. Several non-profit and foundation sponsors provide a broad base of funding support, removing the potential for undue influence by any single organization.

Although the I-SPY 2 TRIAL offered an innovative new system model to develop drugs more efficiently, with real potential to reduce cost, time, and number of study participants required to bring a new therapy to patients, the broad engagement across industry presented a number of challenges. To fully engage pharmaceutical and biomarker companies in this type of precompetitive collaboration, a clear path for drug registration and companion diagnostic (biomarker) approval needed to be defined. While some participating companies wished to use the I-SPY 2 TRIAL process to quickly learn about specific drug pathways and targets, it became clear over the course of engaging partners that if the proof-of-concept phase 2 process was linked to a new regulatory path, there would be broader enthusiasm for the I SPY 2 approach. Therefore, a specific focus of the May 2011 workshop was to explore potential regulatory paths for the evaluation and regulatory review of biomarker-drug combinations that derive from I-SPY 2 and similar trials.

\section{A new clinical trial and regulatory path for drug-companion diagnostics (biomarkers)}

The I-SPY 2 TRIAL was designed as a screening trial, which means its objective is to identify active investigational drugs, potentially paired with biomarkers predictive of response to the particular drug or regimen among high-risk breast cancer patients. Assuming the trial can identify promising regimens, one question raised in the workshop was, what is the most efficient way forward to generate the evidence needed for regulatory review and approval in this setting? Workshop participants proposed the use of pathologic 
complete response (pCR) (in the context of a screening phase 2 trial) as a potentially acceptable biomarker for accelerated approval. The FDA representatives concurred that, in patients with high risk biology (predicted to have early recurrence), pCR meets the definition of an endpoint "that is reasonably likely to predict a clinical outcome" [13] . In I-SPY 2, all patients are profiled and only those who are Her2+, triple negative, or hormone-positive and 70-gene high-risk are randomized. In I-SPY 2, the bar was set fairly high. Success is defined as a doubling of the log odds of pCR, so drugs that graduate from I SPY 2 should have an $85 \%$ predicted probability of success in a confirmatory neoadjuvant trial of 300 patients.

For drugs that appear to significantly increase the rate of pCR within the I-SPY 2 TRIAL screening program, FDA members at the workshop felt that an expedited drug development guidance document using $\mathrm{pCR}$ for accelerated approval could be developed [9]. Draft guidance has now been released [9], and the FDA is in the process of a meta-analysis of neoadjuvant trials and will determine whether three-year or five-year diseasespecific survival is the appropriate endpoint for full approval.

This new draft guidance now provides a regulatory path to obtain accelerated approval for neoadjuvant trials (Figure 3) using pCR as a clinical endpoint. In the proposed guidance, assuming that a well-defined biomarker or companion diagnostic is available to select patients for trials, then a standard single phase 3 trial can be conducted (Figure 3). Alternatively, a drug without a clearly defined companion diagnostic and one that has only completed phase 1 testing might best be tested in a screening phase 2 trial like I-SPY 2, which would allow optimal identification of the patient populations most likely to benefit. This screening phase could then be followed by a second confirmatory neoadjuvant trial.

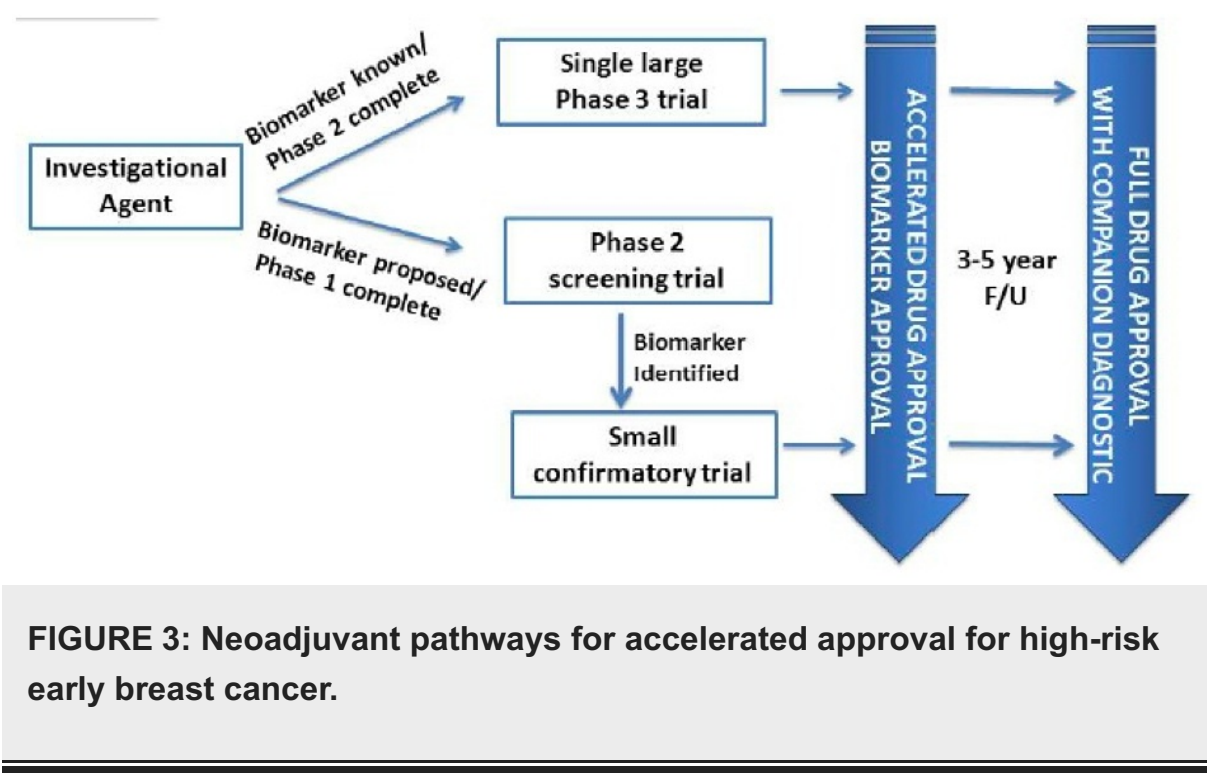

This example of the potential power of the I-SPY 2 TRIAL model highlights the comprehensive and "macro system" view that it provides, a view that should make it possible to reduce knowledge turns and accelerate the time table for getting highly active models to patients. This model represents substantial progress in terms of screening phase 2 drugs, and sets the stage for significantly improving phase 3 trials.

\section{Extending the model and applying it to other diseases}

To achieve the goal of quickly providing new agents to patients, steps prior to the screening phase 2 trial (ISPY 2), such as phase 1 combinations with the standard treatment backbone, and subsequent steps, such as confirmatory testing (i.e., Phase 3), and larger studies to confirm safety, could all use the same efficient, integrated, and collaborative model. Doing so promises to transition a drug candidate seamlessly from the trial screening phase to a broadened Phase 2/3 randomized trial. Another tier 3 alignment process would be to predefine the process for access to agents after accelerated approval, and to link access to collection of safety and outcome data.

Several other diseases might benefit from an I-SPY like approach to developing consortia to collect and share biomarker data tied to clinical outcomes, testing new approaches using an adaptive design, and applying the three-tier model to rapidly evaluate agents. Some examples include other cancers for which there are emerging predictive markers and poor outcomes, such as oligodendroglioma, where $1 \mathrm{p} 19 \mathrm{q}$ codeletion and a neoadjuvant approach may facilitate more rapid identification of agents that improve response [14]. Other diseases include cystic fibrosis, pulmonary fibrosis, autoimmune diseases and potentially Alzheimer's disease. For diseases without acceptable biomarkers that are reasonable reliable early indictors of disease response, the first task would be to form a consortium to redesign the clinical care process to enable integration of the collection of blood and tissue-based markers (ideally, as a routine of the 
course of care), and identify potential markers of the intended outcome. This is essentially the process pursued in the initial testing that established the foundation for the I-SPY I TRIAL [15-17].

In summary, the current process for drug development is too expensive, risky, and inefficient. This process was not designed to test the volume of agents and agent combinations that will result from the rapid expansion of knowledge from the genomic and molecular sciences; realizing the much-anticipated vision of molecular-based personalized medicine will surely take decades under the current structure.

An alternative is the novel three-tier model represented by the I-SPY 2 TRIAL in breast cancer, which incorporates the design of core processes and new structural tools to accommodate these core processes, and achieves better alignment among stakeholders, thereby increasing the potential for successful outcomes. This model contains the key elements of replicable high-functioning approach that is scalable and likely to be applicable across many diseases. Furthermore, this model provides the opportunity for input into potential new paths for regulatory review and accelerated approval of new biomarker/drug combinations performed in the context of I-SPY 2 and I-SPY 2-like trials. Recent draft guidance from the FDA captures this new regulatory path.

\section{Conclusions}

N/A

\section{Appendices}

\section{Additional details regarding the I-SPY 2 TRIAL}

Primary breast cancer, like many diseases, is heterogeneous effectively resulting in patients with a range of risks for progression. For lower-risk patients, the key is to avoid over treatment; while for patients with higher risk of metastatic progression, effective systemic therapy is key to improving survival. While traditionally systemic therapy is given after surgery, studies have show that for high-risk women starting with systemic therapy prior to surgical removal of the tumor does not impact the effectiveness of therapy [9, 10]. Leaving the tumor intact while delivering systemic therapy (neoadjuvant therapy) permits assessment of response early in the course of care and enables the integration of imaging data and tissue collection for biomarkers prior to, during and after treatment. This approach is the genesis of the I-SPY studies.

The I SPY 1 TRIAL was a protocol across nine clinical centers, to demonstrate we could collaborate and acquire biomarkers in the course of standard neoadjuvant care. It set standards for tissue collection, imaging and data acquisition as well as data sharing, and determined how early endpoints, such as volume reduction measured by MRI, and tumor shrinkage or pathologic response assessed at surgery related to long-term outcome as measured by recurrence free survival (RFS) [11]. Pathologic complete response, pCR, or absence of invasive tumor in the breast and lymph nodes at definitive surgery is a good predictor of RFS, but is much stronger when analyzed within tumor subsets [16, 17]. Molecular classifiers identified low risk patients who did not respond to chemotherapy, but had excellent early survival (no early recurrence). Thus, I-SPY 1 also helped to define the selection process and criteria for the population at risk for early recurrence.

The I-SPY 2 TRIAL was designed to introduce novel phase 2 agents into care in the neoadjuvant setting, at a time when women are more curable. The goal of the study is to reduce the time, cost, and number of patients required to identify targeted agents that can increase survival for women with high risk disease and to get such agents to market as quickly as possible. The recruitment is focused on patients with high risk for early recurrence. An FDA approved molecular classifier of risk of progression (70 gene high risk) is used to determine which patients should proceed to randomization [13]. MRI volume, demonstrated to correlate with pCR in the I-SPY 1 TRIAL [15], is measured during neaodjuvant therapy and thus is integrated throughout the course of therapy and used to inform randomization. Thus, imaging guidance from molecular testing is not merely suggested as part of correlative science, it is required and an integral part of the trial.

I-SPY 2 employs an adaptive design in the Phase II setting to efficiently identify agents with a large signal and an $85 \%$ predicted probability of success in the context of a neoadjuvant phase 3 trial in patients with specific tumor subtype(s). Investigational agents, with appropriate safety data, and in combination with paclitaxel, can be tested sequentially in the adjuvant/neoadjuvant setting. Standard chemotherapy, which leads to a pCR in approximately $27 \%$ of patients, is used as the "backbone" and standard arm of the trial. Agents can be moved in and out of the trial seamlessly, with a simple trial amendment as a planned and recurring process.

The I-SPY adaptive design enables a rapid, iterative, "learn as the trial proceeds" approach: If a drug performs better than expected, it can move to confirmatory trials earlier. Drugs that perform less well can be dropped sooner. Unacceptable toxicity will be noted early and could lead to drug removal. The adaptive design also facilitates the identification of the heterogeneity of tumor response as predicted by biomarkers. As the trial proceeds, patients whose tumor biomarkers correlate with a high likelihood of response to a specific drug will be more likely to be randomized to that agent. 
A rapid, iterative, "learn as you go" approach, dictates the need for seamless real-time data collection and analysis, and informatics integration (structural process). Prior to opening the trial, thousands of simulations were run and the threshold for successful/unsuccessful graduation of an agent was predetermined. This formed the basis of the randomization engine. In the I-SPY 2 TRIAL, the impact of a taxane/novel agent(s) combination across 10 biomarker signatures, based on response to therapy as measured by magnetic resonance imaging (MRI volume change), and pathologic extent of disease at the time of definitive surgical treatment continue to inform the randomization.

The complex nature of the I-SPY 2 TRIAL required a more efficient execution strategy than available in normal trial models. Therefore, streamlined infrastructures that provide for multiple biomarkers, drugs, and evaluations at multiple sites, all under central, standardized operational processes, were developed. Currently, the I-SPY-2 trial has been approved at 19 sites with three additional sites pending. Tools used to increase efficiency include:

1) A Master IND in which up to eight drugs can be evaluated at once under a common IND.

2) Early collaborations were established with Local site Institutional Review Boards (IRBs) to educate members on the operational requirements needed to support I-SPY 2's adaptive design and to solicit their input to improve the protocol and process.

3) Testing of multiple levels of biomarkers occurs simultaneously, with three levels of biomarkers under evaluation, including standard (FDA approved biomarkers or IDE based biomarkers for randomization); qualifying biomarkers (performed in a CLIA laboratory); and exploratory biomarkers [14].

4) The FNIH (Foundation of the National Institutes of Health) Biomarker Consortium serves as a trusted broker for a shared intellectual property (IP) agreement, with the same rules applying to all partners across industry, academia, and the community.

5) Quality assurance and improvement are assured through the integration of metrics at all levels of the trial to measure and promote efficiency.

6) Data sharing is assured through submission to_caINTEGRATOR, an NCI-developed database that integrates data from multiple sources, to enable data sharing across the community - with broad data availability six months after a drug leaves the trial.

\section{Additional Information \\ Disclosures}

Conflicts of interest: In compliance with the ICMJE uniform disclosure form, all authors declare the following: Payment/services info: All authors have declared that no financial support was received from any organization for the submitted work. Financial relationships: David Parkinson declare(s) an alternate financial activity from New Enterprise Associates. Employment or leadership position. David M. King Jolly declare(s) an alternate financial activity from Quintiles. Employment or leadership position. Robert Patterson declare(s) an alternate financial activity from Mondobiotech Holdings AG. Employment or leadership position; ownership. Donald A. Berry declare(s) an alternate financial activity from Berry Consultants. Employment or leadership position; ownership. Laura van 't Veer declare(s) an alternate financial activity from Agendia. Employment or leadership position; ownership. John Park declare(s) an alternate financial activity from Merrimack Pharmaceuticals. Consultant or advisory role; ownership. Eric Rubin declare(s) an alternate financial activity from Merck \& Co. Employment or leadership position; ownership. David Parkinson declare(s) an alternate financial activity from Abbott Pharmaceuticals. Consultant or advisory role. Other relationships: All authors have declared that there are no other relationships or activities that could appear to have influenced the submitted work.

\section{References}

1. Centers for Medicare and Medicaid Services, 0.o.t.A., National Health Statistics Group National Health Expenditure Fact Sheet. (2011). Accessed: October 2012:

2. Warren JL, Yabroff KR, Meekins A, et al.: Evaluation of Trends in the Cost of Initial Cancer Treatment . Journal of the National Cancer Institute. 2008, 100:888-897.

3. Wittes RE: Cancer weapons, out of reach. Washington Post. 2004. June 15:

4. DiMasi, J.A., Grabowski, H.G.: Economics of new oncology drug development . Journal of Clinical Oncology. 2007, 25:209.

5. Mullin R: Drug development costs about \$1.7 billion. Chem Eng News. 2003, 81:50.

6. Adams, C.P., Brantner, V.V: Estimating the cost of new drug development: Is it really $\$ 802$ million? . Health Aff (Millwood). 2006, 25:420-28.

7. Dilts DM, Cheng SK, Crites JS, et al.: Phase III clinical trial development: a process of chutes and ladders . Clinical Cancer Research. 2010, 16:5381-89.

8. Kola, I: The state of innovation in drug development . Clinical Pharmacology \& Therapeutics. 2008, 83:227230. 


\section{Cureus}

9. Esserman, L.J, Woodcock, J: Accelerating identification and regulatory approval of investigational cancer drugs. JAMA. 2011, 306:2608-9.

10. Bio/BioMedTracker: Clinical Trial Success Rates Study . (2011). Accessed: October 2012:

http://insidebioia.files.wordpress.com/2011/02/bio-ceo-biomedtracker-bio-study-handout-final-2-15-20.

11. Roberts, S.A., Allen, J.D., Sigal, E.V: Despite criticism of the FDA review process, new cancer drugs reach patients sooner in the United States than in Europe. Health affairs. 2011, 30:1375-81.

12. Grove, A.S: Efficiency in the health care industries. JAMA. 2005, 294:490-2.

13. Prowell, T.M., Pazdur R: Pathological complete response and accelerated drug approval in early breast cancer. N Engl J Med. 2012, 366:2438-41.

14. Galanis E, Wu W, Sarkaria J, et al.: Incorporation of Biomarker Assessment in Novel Clinical Trial Designs: Personalizing Brain Tumor Treatments. Current Oncology Reports. 2011, 13:42-49.

15. Hylton NM, Blume JD, Bernreuter WK, et al.: Locally Advanced Breast Cancer: MR Imaging for Prediction of Response to Neoadjuvant Chemotherapy--Results from ACRIN 6657/I -SPY TRIAL. Radiology. 2012, 263:663-672.

16. Esserman LJ, Berry DA, Cheang MC, et al.: Chemotherapy response and recurrence-free survival in neoadjuvant breast cancer depends on biomarker profiles: results from the I-SPY 1 TRIAL (CALGB 150007/150012; ACRIN 6657). Breast Cancer Res Treat. 2012, 132:1049-62.

17. Esserman LJ, Berry DA, DeMichele A, et al.: Pathologic Complete Response Predicts Recurrence-Free Survival More Effectively by Cancer Subset: Results From the I-SPY 1 TRIAL-CALGB 150007/150012, ACRIN 6657. J Clin Oncol. 2012, 30:3242-9. 